\title{
Canonical structure and extra mode of generalized unimodular gravity
}

\author{
Rodrigo Bufalo ${ }^{1, *}$ and Markku Oksanen ${ }^{2, \dagger}$ \\ ${ }^{1}$ Departamento de Física, Universidade Federal de Lavras, Caixa Postal 3037, \\ 37200-000 Lavras, Minas Gerais, Brazil \\ ${ }^{2}$ Department of Physics, University of Helsinki, P.O. Box 64, FI-00014 Helsinki, Finland
}

(Received 11 January 2018; published 9 February 2018)

\begin{abstract}
We consider a recently proposed generalization of unimodular gravity, where the lapse function is constrained to be equal to a function of the determinant of the spatial metric $f(h)$, as a potential origin of a dark fluid with a generally $h$-dependent equation of state parameter. We establish the Hamiltonian analysis and the canonical path integral for the theory. All the special cases that do not match unimodular gravity involve the violation of general covariance, and consequently the physical content of the theory is changed significantly. Particularly, the case of a constant function $f$ is shown to contain an extra physical degree of freedom in each point of space. Physical consequences of the extra degree of freedom are studied in a linearized theory, where the extra mode is carried by the trace of the metric perturbation. The trace mode does not propagate as a wave, since it satisfies an elliptic partial differential equation in spacetime. Consequently, the trace perturbation is shown to grow exponentially with time, which implies instability. The case of a general $f(h)$ involves additional second-class constraints, which implies the presence of an extra global degree of freedom that depends only on time (instead of the extra local degree of freedom in the case of a constant $f$ ).
\end{abstract}

DOI: 10.1103/PhysRevD.97.044014

\section{INTRODUCTION}

Despite the great progress on experimental and theoretical understanding in modern cosmology, we still face difficulties in three major issues: dark matter, dark energy, and the cosmological constant problem. In view of the standard model of cosmology, many minimal modifications of general relativity (GR) have been proposed and explored in order to attempt to understand the fundamental origin of one or more of the above problems. The most used approach for the description of such phenomena involves the addition of new (global and/or local) degrees of freedom. Instead of adding new fields, an appealing way to incorporate new degrees of freedom in this context is by enforcing a symmetry principle.

One of the simplest modifications of GR that has been used to elucidate the cosmological constant problem is unimodular gravity $[1,2]$. In unimodular gravity, general covariance is restricted to diffeomorphisms which preserve the determinant of the metric of spacetime. It is reasonable to say that, at the classical level, the main conceptual difference compared to GR is that the cosmological

\footnotetext{
*odrigo.bufalo@dfi.ufla.br

†markku.oksanen@helsinki.fi
}

Published by the American Physical Society under the terms of the Creative Commons Attribution 4.0 International license. Further distribution of this work must maintain attribution to the author(s) and the published article's title, journal citation, and DOI. Funded by SCOAP. constant in unimodular gravity is a constant of integration rather than a coupling constant $[2-5] .{ }^{1}$ Although it was initially expected that this different point of view could shed new light on the cosmological constant problem, a similar problem with the fine-tuning of the cosmological constant is found as in GR [6].

Based on the key concepts of unimodular gravity, a new proposal, namely, vacuum energy sequestering [7], has been presented as a mechanism for providing a radiatively stable cosmological constant, which is independent of the vacuum energy contributions from the matter sector. The main idea of this mechanism is to impose a global scaling symmetry, which complements unimodular gravity by introducing a variational procedure that fixes the values of global variables so that the cosmological constant is decoupled from the vacuum energy generated by matter loop corrections. This is achieved by the addition of (global) conserved quantities into the gravitational action, which provide a finite value for the cosmological constant and at the same time cancel out all quantum-generated vacuum energy contributions of the matter sector from the gravitational equations of motion. In order to explain the microscopic origin of the sequestering mechanism, a local formulation of the theory has been proposed [8]. Actually, the local setup is obtained from the global one by using a similar reparametrization invariance

\footnotetext{
${ }^{1}$ This fact is also present in a path integral analysis, where the value of the cosmological constant $\Lambda$ is included in the initial and boundary conditions and is not present as a coupling constant in the Lagrangian [5]
} 
approach as in the Henneaux-Teitelboim formulation of unimodular gravity [9].

On the other hand, instead of adding scaling and reparametrization invariance into unimodular gravity in order to secure a finite and stable cosmological constant, other interesting modifications of the symmetries of GR have been considered for the description of different physical phenomena. For example, a restriction to foliation-preserving diffeomorphism at high energies (in the ultraviolet fixed point) has been considered as a way to solve the renormalizability and ghost problems of the quantum field theory of gravity [10]. A conformally invariant extension of GR has been shown to include a gravitational degree of freedom that mimics dark dust [11]. A new example of such models has been recently proposed, where, instead of enlarging the group of symmetry, a certain type of Lorentz violation is incorporated into unimodular gravity in order to induce a dark fluid [12]. This theory is referred to as generalized unimodular gravity. A breakdown of (gauge) spacetime symmetry is a wellknown approach to enlarge the physical content of a theory. The chosen breaking of general covariance is defined in terms of the Arnowitt-Deser-Misner (ADM) decomposition of the metric [13]. The unimodular constraint $\sqrt{-g}=\epsilon_{0}$, where $\epsilon_{0}$ is fixed, is replaced with

$$
N=f(h),
$$

where $N=\left(-g^{00}\right)^{-1 / 2}$ is the lapse function and $f(h)$ is a function of the determinant $h$ of the induced metric $h_{i j}$ on the spatial hypersurfaces $\Sigma_{t}$ of the foliation of spacetime. This can be seen as a generalization of the unimodular constraint, since (1) is equivalent to

$$
\sqrt{-g}=\sqrt{h} f(h) .
$$

The motivation for this generalization is twofold [12]: a minimal breakdown of Lorentz symmetry $O(1,3)$ to $O(3)$ and the presence of a special type of matter source at the classical level, a general barotropic dark fluid with an equation of state parameter that depends on the metric determinant $h$.

Unimodular gravity is included in the generalized theory as the special case $f(h)=\epsilon_{0} / \sqrt{h}$. In the special case $f(h)=$ const, the engendered dark fluid behaves as a dust, which, in principle, could describe pressureless dark matter. However, care must be paid to the nature of the Lagrange multiplier that is used to enforce the generalized unimodular constraint in the action. Actually, this field can be seen either as an undetermined variable, which can be eliminated, or as an extra energy density for the Einstein equation. Both interpretations describe the same physical system, but due to subtleties along the analysis of the field equations it is always possible to overlook constraints among the variables and then obtain an erroneous result. This is carefully examined in Sec. II.

A clear understanding of the nature and conclusive counting of the physical degrees of freedom can unambiguously be obtained from a canonical analysis of the theory rather than from the equations of motion. Hence, the main goal for the present work is to perform a Hamiltonian analysis of the generalized unimodular gravity for any Lorentz-violating function $f(h)$, in order to have a complete understanding of the physical content of the model. The paper is organized as follows. In Sec. II, we present the generalized unimodular gravity and its symmetry content, elucidating the implications of the Lorentz violation into the field equations and the subtleties involved in the presence of the dark fluid, particularly regarding the interpretation of the Lagrange multiplier field $\lambda$ as a genuine variable or as an energy density. Section III is dedicated to the Hamiltonian analysis of the generalized model. We determine the canonical structure for some special case of the function $f(h)$ and show how the number and nature of constraints, and consequently the number of physical degrees of freedom, are changed compared to GR and (customary) unimodular gravity. In Sec. IV, the canonical path integral is established for the special case $f=$ const and general $f(h)$, highlighting the difference in their physical content, i.e., degrees of freedom. In Sec. V, we consider a linearization of the generalized theory in order to examine the dynamics of the extra physical degree of freedom. Final remarks are presented in Sec. VI.

\section{GENERALIZED UNIMODULAR GRAVITY}

The action for generalized unimodular gravity can be defined by adding the constraint (1) into the EinsteinHilbert action by means of a Lagrange multiplier $\lambda$ [12]:

$S\left[g_{\mu \nu}, \lambda\right]=\int d^{4} x\left[\frac{M_{\mathrm{P}}^{2}}{2} \sqrt{-g} R-\lambda\left(\frac{1}{\sqrt{-g^{00}}}-f(h)\right)\right]$.

Matter fields are coupled to the metric in the usual way.

We rewrite the full action for the generalized unimodular theory of gravity as

$$
\begin{aligned}
S\left[g_{\mu \nu}, \lambda, \Psi\right]= & \int d^{4} x\left[\frac{M_{\mathrm{P}}^{2}}{2} \sqrt{-g} R-\lambda(\sqrt{-g}-\sqrt{h} f(h))\right] \\
& +S_{\mathrm{m}}\left[g_{\mu \nu}, \Psi\right],
\end{aligned}
$$

where matter fields are denoted by $\Psi$. The omitted boundary terms of the action are the same as in GR [14], as well as in unimodular gravity [5]. The action (4) differs from the one (3) proposed in Ref. [12] only by the nature of the Lagrange multiplier field $\lambda$. In (3), the Lagrange multiplier is a scalar density of unit weight on $\Sigma_{t}$. Our $\lambda$ in (4), on the other hand, is a scalar field on spacetime and of course on $\Sigma_{t}$ as well. As a result, the first 
term of the constraint part of the action (4) is generally invariant, while the second term breaks general covariance. The difference to conventional formulations of unimodular gravity [5] appears in the second term of the constraint part. Compared to the action of unimodular gravity with a fixed metric determinant, $\sqrt{-g}=\epsilon_{0}$ (see [5]), the fixed scalar density $\epsilon_{0}$ has been replaced with a function of the spatial metric determinant as $\sqrt{h} f(h)$. Generalized unimodular gravity reduces to the traditional theory when the function $f$ is chosen as $f(h)=\epsilon_{0} / \sqrt{h}$. We emphasize that the inclusion of the constraint (2) in the action (4) is not equivalent to imposing the constraint as a gauge-fixing condition in GR, since the Hamiltonian constraints in the given two theories are different. Therefore, the constraint (2) has physical consequences. The number of physical degrees of freedom is shown to depend on the choice of the function $f$. For instance, in the case of a constant function $f$, the Hamiltonian constraint becomes a second-class constraint, which is used to determine the variable $\lambda$. Then there is one less constraint on the metric, and hence an extra physical degree of freedom is present.

In the general $f(h)$ case, the symmetry under diffeomorphisms is restricted as follows. Consider an infinitesimal diffeomorphism generated by $\xi^{\mu}$ :

$$
\delta_{\xi} g_{\mu \nu}=\nabla_{\mu} \xi_{\nu}+\nabla_{\nu} \xi_{\mu} .
$$

According to (2), the action is invariant under (5) if the diffeomorphisms are restricted by

$$
\delta_{\xi} \sqrt{-g}=\delta_{\xi}(\sqrt{h} f(h)),
$$

which holds when $\xi^{\mu}$ satisfies the condition

$$
\nabla_{\mu} \xi^{\mu}=N^{-1}\left(f(h)+2 h f^{\prime}(h)\right) h^{i j} \nabla_{i} \xi_{j},
$$

where $f^{\prime}(h)=d f(h) / d h$ and $\xi_{i}=g_{i \mu} \xi^{\mu}, i=1,2$, 3. In the special case of unimodular gravity, we obtain the metric determinant-preserving diffeomorphisms, $\delta_{\xi} \sqrt{-g}=$ $0 \Rightarrow \nabla_{\mu} \xi^{\mu}=0$.

The field equation obtained by varying $\lambda$ is precisely (2) or equivalently (1), and the field equations for matter are identical to those in GR. The field equations obtained by varying the action (4) with respect to $g^{\mu \nu}$ is

$$
G_{\mu \nu}=M_{\mathrm{P}}^{-2}\left(T_{\mu \nu}+\tau_{\mu \nu}\right),
$$

where $G_{\mu \nu}$ is the Einstein tensor, $T_{\mu \nu}$ is the usual stressenergy tensor of matter, $T_{\mu \nu}=-\frac{2}{\sqrt{-g}} \frac{\delta S_{\mathrm{m}}}{\delta g^{m \nu}}$, and the stressenergy tensor of the additional (dark) fluid is written as

$$
\tau_{\mu \nu}=-\lambda g_{\mu \nu}+\lambda N^{-1}\left(f(h)+2 h f^{\prime}(h)\right) h_{\mu \nu} .
$$

Here $h_{\mu \nu}$ is the metric induced by $g_{\mu \nu}$ onto the spatial hypersurface $\Sigma_{t}$ :

$$
h_{\mu \nu}=g_{\mu \nu}+n_{\mu} n_{\nu}
$$

where $n_{\mu}$ is the unit normal to $\Sigma_{t}$ :

$$
n_{\mu}=-N \nabla_{\mu} t=-N \delta_{\mu}^{0} .
$$

The stress-energy tensor (9) can be written in the form of a perfect fluid with a velocity $n_{\mu}$ :

$$
\tau_{\mu \nu}=(\rho+p) n_{\mu} n_{\nu}+p g_{\mu \nu},
$$

where the energy density $\rho$ and the pressure $p$ are identified, respectively, as

$$
\rho=\lambda, \quad p=-\lambda+\lambda N^{-1}\left(f(h)+2 h f^{\prime}(h)\right) .
$$

When the constraint (1) is satisfied, the pressure reduces to

$$
p=\lambda \frac{2 h f^{\prime}(h)}{f(h)} .
$$

Thus, the dark fluid satisfies the equation of state $p=w \rho$ with a parameter $w$ that generally depends on the determinant of the spatial metric as

$$
w(h)=\frac{2 h f^{\prime}(h)}{f(h)} .
$$

In the case of a constant function $f$, we have dark dust with energy density $\lambda$ and no pressure $(w=0)$.

While the appearance of the dark fluid (12) in the stressenergy tensor is evident, it is crucial to acknowledge that the energy density $\lambda$ is arbitrary. Although the field $\lambda$ is not a dynamical variable, in the sense of not having a field equation, it cannot be fixed at will either, since we considered $\lambda$ to be a genuine variable of the action in order to impose the generalized unimodular condition (1). This suggests that there is an extra physical degree of freedom in the theory due to the presence of the variable $\lambda$, which is not carried by this scalar field, since we have no dynamical field equation for it. When such a nondynamical variable is present in the action, we can always attempt to eliminate it by using a field equation that involves the variable. In this case, the relevant equation is the full projection of the modified Einstein equation (8) perpendicular to $\Sigma_{t}^{2}$ :

$$
G_{n n}=M_{\mathrm{P}}^{-2}(\mathcal{E}+\lambda),
$$

\footnotetext{
${ }^{2}$ The projection of the Einstein tensor along the unit normal $n^{\mu}$ is written in terms of the intrinsic scalar curvature ${ }^{(3)} R$ and extrinsic curvature $K_{i j}$ of the spatial hypersurface $\Sigma_{t}$ as

$$
G_{\mu \nu} n^{\mu} n^{\nu}=\frac{1}{2}\left({ }^{(3)} R+K^{2}-K_{i j} K^{i j}\right) .
$$
}


where we denote $G_{n \boldsymbol{n}}=G_{\mu \nu} n^{\mu} n^{\nu}$ and $\mathcal{E}=T_{\mu \nu} n^{\mu} n^{\nu}$. $\mathcal{E}$ is the energy density of matter measured by an Eulerian observer with four-velocity $n^{\mu}$, i.e., an observer comoving with the dark fluid. Since $\lambda$ is arbitrary and not measurable, it is appropriate to regard that Eq. (16) determines $\lambda$, rather than determining the given projection of the Einstein tensor for an energy density $\mathcal{E}+\lambda^{3}{ }^{3}$ Therefore, we regard that $\lambda$ is determined by the projection $G_{n n}$ of the Einstein tensor and the energy density of matter as

$$
\lambda=M_{\mathrm{P}}^{2} G_{n n}-\mathcal{E} .
$$

That is inserted back into the remaining projections of the modified Einstein equation, namely, to the full projection of (8) onto $\Sigma_{t}$ and to the mixed projection of (8) onto $\Sigma_{t}$ and $n^{\mu}$.

In the case of a constant $f$, the field equation (8) is rewritten using (17) as

$$
G_{\mu \nu}-G_{n \boldsymbol{n}} n_{\mu} n_{\nu}=M_{\mathrm{P}}^{-2}\left(T_{\mu \nu}-\mathcal{E} n_{\mu} n_{\nu}\right) .
$$

This is the Einstein equation with its projection perpendicular to $\Sigma_{t}$ subtracted. Since there is now one equation less to determine the gravitational field than in GR, consequently there should appear an extra physical degree of freedom in the gravitational sector.

The case of a general function $f$ can be analyzed in a similar way. However, the field equation is more involved:

$$
\begin{aligned}
G_{\mu \nu} & -G_{n n}\left(n_{\mu} n_{\nu}+\frac{2 h f^{\prime}(h)}{f(h)} h_{\mu \nu}\right) \\
= & M_{\mathrm{P}}^{-2}\left[T_{\mu \nu}-\mathcal{E}\left(n_{\mu} n_{\nu}+\frac{2 h f^{\prime}(h)}{f(h)} h_{\mu \nu}\right)\right],
\end{aligned}
$$

so that it is less evident how many independent equations exist for the gravitational field. Since the full projection perpendicular to $\Sigma_{t}$ still vanishes trivially, we can expect an increase in the number of degrees of freedom (at least globally).

On the other hand, an alternative approach to the field equations is to keep $\lambda$ and begin to regard the dark fluid as a true additional matter source in the Einstein equation (8). Essentially, the field $\lambda$ would no longer be a regular variable of the gravitational theory, and instead we begin to consider it as the energy density of an additional perfect fluid (12). Then the dark fluid behaves as any perfect fluid with energy density $\lambda$ and ( $h$-dependent) pressure (14). Assuming that the stress-energy tensor of normal matter is conserved, $\nabla^{\nu} T_{\mu \nu}=0$, we may take the divergence of the modified Einstein equation (8), so that the stress-energy tensor of the dark fluid (12) must be conserved as well, $\nabla^{\nu} \tau_{\mu \nu}=0$. In unimodular gravity, this gives $\nabla_{\mu} \lambda=0$, which means that $\lambda$

\footnotetext{
${ }^{3}$ Actually, when $\lambda$ is unknown, Eq. (16) cannot be used to find $G_{n n}$, since the source in the right-hand side of the equation is undetermined.
}

is a constant, namely, the cosmological constant. In the present generalized theory, we obtain a more involved conservation equation as

$$
\begin{aligned}
& \left(\nabla_{n}+K\right)[\lambda(1+w(h))] n_{\mu}+\nabla_{\mu}[\lambda w(h)] \\
& \quad+\lambda(1+w(h)) a_{\mu}=0,
\end{aligned}
$$

where $\nabla_{n}=n^{\mu} \nabla_{\mu}, K$ is the trace of the extrinsic curvature of the hypersurface $\Sigma_{t}$, and $a_{\mu}=n^{\nu} \nabla_{\nu} n_{\mu}$ is the acceleration of an Eulerian observer. The projections of (20) along $n^{\mu}$ and onto $\Sigma_{t}$ are written as

$$
\begin{gathered}
\nabla_{n} \lambda+\lambda(1+w(h)) K=0, \\
\partial_{i}[\lambda w(h)]+\lambda(1+w(h)) a_{i}=0,
\end{gathered}
$$

where $\nabla_{n} \lambda=\frac{1}{N}\left(\partial_{t} \lambda-N^{i} \partial_{i} \lambda\right)$ and we have assumed that $f(h)$ behaves as a scalar on the spatial hypersurface, so that the pressure behaves as a scalar as well, and consequently its covariant derivative on the spatial hypersurface reduces to a partial derivative, $h^{\mu}{ }_{i} \nabla_{\mu}[\lambda w(h)]=D_{i}[\lambda w(h)]=\partial_{i}[\lambda w(h)]$. These equations can be solved for $\lambda$ with appropriate boundary conditions. In the case of a constant $f$, the conservation equations have the usual form for a dust:

$$
\nabla_{n} \lambda+K \lambda=0, \quad \lambda a_{i}=0 .
$$

The trivial solution of a constant $\lambda$ for these equations is permitted only if $K=0$. When $K \neq 0, \lambda$ is a nontrivial solution to the first equation, and the second equation becomes $a_{i}=0$.

While the analysis of generalized unimodular gravity can be achieved at the level of field equations for any function $f$, as described above, there is a risk of overlooking constraints among the variables. Thus, we shall perform a Hamiltonian analysis of the theory, which will reveal all the constraints and the structure of the gauge symmetry. Moreover, the canonical analysis will provide a conclusive counting and the physical nature of the degrees of freedom.

\section{HAMILTONIAN ANALYSIS}

\section{A. Hamiltonian and constraints}

The gravitational part of the action (4) is written in terms of $\mathrm{ADM}$ variables as

$$
\begin{aligned}
S_{g}\left[N, N^{i}, h_{i j}, \lambda\right]= & \int d t \int_{\Sigma_{t}} d^{3} x \sqrt{h}\left[\frac { M _ { \mathrm { P } } ^ { 2 } } { 2 } N \left(K_{i j} \mathcal{G}^{i j k l} K_{k l}\right.\right. \\
& \left.\left.+{ }^{(3)} R\right)-\lambda(N-f(h))\right]
\end{aligned}
$$

where $K_{i j}$ is the extrinsic curvature of the spatial hypersurface $\Sigma_{t}$, 


$$
K_{i j}=\frac{1}{2 N}\left(\partial_{t} h_{i j}-2 D_{(i} N_{j)}\right)
$$

the De Witt metric is defined as

$$
\mathcal{G}^{i j k l}=\frac{1}{2}\left(h^{i k} h^{j l}+h^{i l} h^{j k}\right)-h^{i j} h^{k l},
$$

and ${ }^{(3)} R$ is the (intrinsic) scalar curvature of $\Sigma_{t}$. We introduce the canonical momenta $\pi_{N}, \pi_{i}, \pi^{i j}$, and $p_{\lambda}$ conjugate to $N, N^{i}, h_{i j}$, and $\lambda$, respectively. Since the action (24) is independent of the time derivatives of the variables $N, N^{i}$, and $\lambda$, their canonically conjugated momenta are primary constraints:

$$
\pi_{N} \approx 0, \quad \pi_{i} \approx 0, \quad p_{\lambda} \approx 0 .
$$

The momentum conjugate to the metric $h_{i j}$ is defined as

$$
\pi^{i j}=\frac{M_{\mathrm{P}}^{2}}{2} \sqrt{h} \mathcal{G}^{i j k l} K_{k l} .
$$

The Hamiltonian is obtained as

$$
\begin{aligned}
H= & \int_{\Sigma_{t}} d^{3} x\left(N \mathcal{H}_{T}+N^{i} \mathcal{H}_{i}-\sqrt{h} \lambda f(h)\right. \\
& \left.+v_{N} \pi_{N}+v_{N}^{i} \pi_{i}+v_{\lambda} p_{\lambda}\right),
\end{aligned}
$$

where the so-called super-Hamiltonian and supermomentum are defined as

$$
\mathcal{H}_{T}=\frac{2}{M_{\mathrm{P}}^{2} \sqrt{h}} \pi^{i j} \mathcal{G}_{i j k l} \pi^{k l}-{\frac{M_{\mathrm{P}}^{2} \sqrt{h}}{2}}^{(3)} R+\sqrt{h} \lambda
$$

and

$$
\mathcal{H}_{i}=-2 h_{i j} D_{k} \pi^{j k}+\partial_{i} N \pi_{N}+\partial_{i} \lambda p_{\lambda}
$$

respectively, where we have introduced the inverse De Witt metric as

$$
\mathcal{G}_{i j k l}=\frac{1}{2}\left(h_{i k} h_{j l}+h_{i l} h_{j k}\right)-\frac{1}{2} h_{i j} h_{k l}
$$

and $v_{N}, v_{N}^{i}$, and $v_{\lambda}$ are unspecified Lagrange multipliers for the primary constraints. The momentum constraint (31) has been extended with terms that are proportional to the primary constraints $\pi_{N}$ and $p_{\lambda}$, so that the variables $N$ and $\lambda$ transform as scalar fields under the spatial diffeomorphisms generated by (31).

The surface terms have been omitted, since we have confirmed that the surface terms and their contribution to the total gravitational energy remain identical to the ones in the cases of GR [14] and unimodular gravity with a fixed metric determinant [5]. For further details, see the discussion in Sec. III D.

Consistency of the primary constraints implies the secondary constraints

$$
\mathcal{H}_{T} \approx 0, \quad \mathcal{H}_{i} \approx 0, \quad \mathcal{C}_{N}=N-f(h) \approx 0 .
$$

The Hamiltonian and momentum constraints satisfy the same Poisson brackets as in GR. The modified unimodular constraint $\mathcal{C}_{N}$ has a nonvanishing Poisson bracket with the Hamiltonian and momentum constraints

$$
\left\{\mathcal{C}_{N}, \int_{\Sigma_{t}} d^{3} x \xi \mathcal{H}_{T}\right\}=\frac{2}{M_{\mathrm{P}}^{2}} \xi \sqrt{h} f^{\prime}(h) h_{i j} \pi^{i j},
$$

$$
\begin{aligned}
\left\{\mathcal{C}_{N}, \int_{\Sigma_{t}} d^{3} x \chi^{i} \mathcal{H}_{i}\right\} & =\chi^{i} \partial_{i} N-\chi^{i} \partial_{i} f(h)-2 \partial_{i} \chi^{i} f^{\prime}(h) h \\
& \approx-2 \partial_{i} \chi^{i} f^{\prime}(h) h .
\end{aligned}
$$

We see that $\mathcal{C}_{N}$ and $\pi_{N} \approx 0$ are necessarily second-class constraints, since

$$
\left\{\mathcal{C}_{N}(x), \pi_{N}(y)\right\}=\delta(x, y) .
$$

The consistency of $\mathcal{C}_{N}$ is ensured by fixing the Lagrange multiplier $v_{N}$ as

$v_{N}=u_{N} \equiv-\frac{2}{M_{\mathrm{P}}^{2}} N \sqrt{h} f^{\prime}(h) h_{i j} \pi^{i j}+2 \partial_{i} N^{i} f^{\prime}(h) h$.

The consistency condition for $\mathcal{H}_{T}$,

$\left\{\mathcal{H}_{T}, H\right\} \approx-\frac{2}{M_{\mathrm{P}}^{2}} \lambda\left(\frac{f(h)}{2}+h f^{\prime}(h)\right) h_{i j} \pi^{i j}+\sqrt{h} v_{\lambda} \approx 0$,

fixes the Lagrange multiplier $v_{\lambda}$ as

$$
v_{\lambda}=u_{\lambda} \equiv \frac{2}{M_{\mathrm{P}}^{2}} \lambda\left(\frac{f(h)}{2}+h f^{\prime}(h)\right) \frac{h_{i j} \pi^{i j}}{\sqrt{h}} .
$$

The Hamiltonian is then written as

$H=\int_{\Sigma_{t}} d^{3} x\left(N \mathcal{H}_{T}^{\prime}+N^{i} \mathcal{H}_{i}^{\prime}-\sqrt{h} \lambda f(h)+v_{N}^{i} \pi_{i}+u_{\lambda} p_{\lambda}\right)$,

where the new Hamiltonian and momentum constraints are defined, respectively, as

$$
\mathcal{H}_{T}^{\prime}=\mathcal{H}_{T}-\frac{2}{M_{\mathrm{P}}^{2}} \sqrt{h} f^{\prime}(h) h_{i j} \pi^{i j} \pi_{N} \approx 0
$$

and

$$
\mathcal{H}_{i}^{\prime}=\mathcal{H}_{i}-2 \partial_{i}\left(h f^{\prime}(h) \pi_{N}\right) \approx 0 .
$$

We now see that the consistency condition for $\mathcal{H}_{i}$,

$$
\left\{\mathcal{H}_{i}, H\right\} \approx-\sqrt{h} \partial_{i}\left[f(h)+2 h f^{\prime}(h)\right] \lambda-2 \sqrt{h} h f^{\prime}(h) \partial_{i} \lambda \approx 0,
$$

requires the postulation of a new constraint 


$$
\mathcal{C}_{i}=\left[3 f^{\prime}(h)+2 h f^{\prime \prime}(h)\right] \partial_{i} h \lambda+2 h f^{\prime}(h) \partial_{i} \lambda \approx 0 .
$$

As we have seen before, there are two important special cases for the generalized theory: $f(h)=$ const and $f(h)=\epsilon_{0} / \sqrt{h}$. These two cases also stand out in the canonical structure of the theory. After these two cases are explained, we shall consider all the other functions $f$.

When $f(h)$ is a constant, the lapse function $N$ is fixed to a constant by the constraint $\mathcal{C}_{N}=N-f \approx 0$. Since $f^{\prime}(h)=0$, the dark fluid of Ref. [12] would have a vanishing equation of state parameter (15), $w=0$, which is the case of dark dust discussed in Ref. [12]. The present canonical analysis shows that this case contains an extra physical degree of freedom in each point of space, which may explain the spatial inhomogeneities of the dark fluid. Now the constraint $\mathcal{C}_{N}$ has a vanishing Poisson bracket with $\mathcal{H}_{T}$ and $\mathcal{H}_{i}$, since $f^{\prime}(h)=0$ in (34) and (35). Furthermore, in this case, the constraint $\mathcal{C}_{i}$ (44) does not appear at all. Hence, we have four second-class constraints: $\mathcal{C}_{N} \approx 0$, $\pi_{N} \approx 0, \mathcal{H}_{T} \approx 0$, and $p_{\lambda} \approx 0$. When the Dirac bracket for the second-class constraints is introduced, and the constraints are imposed strongly, we can eliminate the variables $N, \pi_{N}, \lambda$, and $p_{\lambda}$. The Dirac bracket can be shown to be equivalent to the Poisson bracket for all the remaining variables. The Hamiltonian is thus obtained as

$$
H=\int_{\Sigma_{t}} d^{3} x\left(f \mathcal{H}_{T}^{0}+N^{i} \mathcal{H}_{i}+v_{N}^{i} \pi_{i}\right),
$$

where the first-class constraints are $\mathcal{H}_{i}=-2 h_{i j} D_{k} \pi^{j k} \approx 0$ and $\pi_{i} \approx 0$, which are associated with the symmetry under spatial diffeomorphisms, and we denote the superHamiltonian without a cosmological constant as

$$
\mathcal{H}_{T}^{0}=\frac{2}{M_{\mathrm{P}}^{2} \sqrt{h}} \pi^{i j} \mathcal{G}_{i j k l} \pi^{k l}-\frac{M_{\mathrm{P}}^{2} \sqrt{h}}{2}{ }^{(3)} R .
$$

Note that $\mathcal{H}_{T}^{0}$ is not a constraint. The constraint $\mathcal{H}_{T}$ served only to determine the variable $\lambda$ as $\lambda=-\mathcal{H}_{T}^{0} / \sqrt{h}$. Since the two terms of the Hamiltonian (40) that involved $\lambda$ canceled out when $N=f(h)$ was imposed, the value of $\lambda$ is irrelevant, and hence the situation is exactly the same as having no Hamiltonian constraint at all. The Hamiltonian (45) is equal to the Hamiltonian of GR with the lapse function fixed to a constant $f$ and without a Hamiltonian constraint. It is interesting to realize that imposing the lapse function to a constant with a constraint multiplied by a Lagrange multiplier field in the action (4) leads to a breakdown of the diffeomorphism invariance all the way down to invariance under spatial diffeomorphism. Moreover, it is worth noticing that the absence of a Hamiltonian constraint implies the presence of an extra physical degree of freedom for each point of space, which is carried by the metric.
When $f(h)=\epsilon_{0} / \sqrt{h}$, we have the case of unimodular gravity, where the constraint $\mathcal{C}_{N}$ is equivalent to $\sqrt{-g}-\epsilon_{0} \approx 0$. More generally, the function $f$ may contain an additional constant $c_{0}$ as $f(h)=\epsilon_{0} / \sqrt{h}+c_{0}$. However, that case would be related to unimodular gravity via a translation of the lapse function. In either case, the constraint (44) is reduced to a simple form: $\partial_{i} \lambda \approx 0$ [5]. Now the spatial gradient of the variable $\lambda$ is constrained to vanish everywhere. The constant value of $\lambda$ is the cosmological constant in unimodular gravity. A complete Hamiltonian analysis of this case is found in Ref. [5]. Classically, this case is equivalent to GR with a cosmological constant. A subtle difference appears at the quantum level, since the value of the cosmological constant is set as a part of the initial conditions and the path integral may be extended to include integration over the cosmological constant [3-5].

When $f^{\prime}(h) \neq 0$ everywhere and $f(h)$ does not match the case of unimodular gravity, i.e., $3 f^{\prime}(h)+2 h f^{\prime \prime}(h) \neq 0$, the constraint (44) imposes a relation between the variables $\lambda$ and $h$, and hence it is much more complicated than the corresponding constraint of unimodular gravity. First we shall rewrite the constraint (44) to a simpler form by multiplying it with $\frac{1}{2} h^{1 / 2}$ and combining the three terms together. Thus, we can redefine the constraint (44) as

$\mathcal{C}_{i}=\frac{1}{\sqrt{h}} \partial_{i}\left(\sqrt{h} F_{1}(h) \lambda\right) \approx 0, \quad F_{1}(h)=h f^{\prime}(h)$.

The factor $h^{-1 / 2}$ in front of (47) ensures that $\mathcal{C}_{i}$ is a scalar constraint rather than a density. We denote $h f^{\prime}(h)$ as $F_{1}(h)$ for the purpose of reminding us that this function shall be treated as a scalar along with $f(h)$ when integrated. Generally, for the $n$th order derivative of $f$ we denote

$$
F_{n}(h)=h^{n} f^{(n)}(h)
$$

Let us return to the canonical analysis, and the consistency condition for $\mathcal{C}_{i}$ can be obtained as $\left(\xi^{i}\right.$ is an arbitrary smearing function)

$$
\begin{aligned}
\left\{\int_{\Sigma_{t}} d^{3} x \sqrt{h} \xi^{i} \mathcal{C}_{i}, H\right\} \approx & \int_{\Sigma_{t}} d^{3} x \partial_{k} \xi^{k}\left(\frac { 2 } { M _ { \mathrm { P } } ^ { 2 } } \left[f(h) F_{1}(h)\right.\right. \\
& \left.-F_{1}^{2}(h)+f(h) F_{2}(h)\right] \lambda h_{i j} \pi^{i j} \\
& -\sqrt{h} N^{i} \mathcal{C}_{i}-\partial_{i} N^{i} \sqrt{h}\left[3 F_{1}(h)\right. \\
& \left.\left.+2 F_{2}(h)\right] \lambda\right),
\end{aligned}
$$

which has to vanish on the constraint surface. The local version of the condition is obtained by integration by parts and by setting $\xi^{k}=h^{-1 / 2}(x) \delta_{i}^{k} \delta(x, z)$. Therefore we need to impose a new constraints as 


$$
\begin{aligned}
\mathcal{C}_{i}^{N}= & \frac{1}{\sqrt{h}} \partial_{i}\left(\partial_{j} N^{j} \sqrt{h}\left[3 F_{1}(h)+2 F_{2}(h)\right]\right. \\
& \left.-\frac{2}{M_{\mathrm{P}}^{2}}\left[f(h) F_{1}(h)-F_{1}^{2}(h)+f(h) F_{2}(h)\right] h_{j k} \pi^{j k}\right) \approx 0 .
\end{aligned}
$$

This constraint can be regarded as a condition on the shift vector $N^{i}$ (or rather on its spatial divergence), and it is a second-order partial differential equation (PDE) for $N^{i}$. The constraint (50) does not constrain the divergence-free component of $N^{i}$. Therefore, we consider a Helmholtz decomposition of the shift vector

$$
N^{i}=N_{1}^{i}+N_{\mathrm{t}}^{i}
$$

where $\partial_{i} N_{\mathrm{t}}^{i}=0$, so that $\partial_{i} N^{i}=\partial_{i} N_{1}^{i}$. We could introduce a scalar potential $\phi$ and a vector potential $A_{i}$ to write the components as

$$
N_{1}^{i}=-\partial^{i} \phi, \quad N_{\mathrm{t}}^{i}=\epsilon^{i j k} \partial_{j} A_{k},
$$

but this is not necessary for our present purposes. The canonical momenta $\pi_{i}$ should be decomposed correspondingly:

$$
\pi_{i}=\pi_{i \mid 1}+\pi_{i \mid \mathrm{t}}
$$

so that the nonvanishing Poisson brackets between the components of (51) and (53) are

$$
\begin{aligned}
& \left\{N_{1}^{i}(x), \pi_{j \mid 1}(y)\right\}=\delta_{j}^{i} \delta(x, y), \\
& \left\{N_{\mathrm{t}}^{i}(x), \pi_{j \mid \mathrm{t}}(y)\right\}=\delta_{j}^{i} \delta(x, y) .
\end{aligned}
$$

Now (50) constrains only the longitudinal component $N_{1}^{i}$, while the transverse component $N_{\mathrm{t}}^{i}$ is left to be determined with a gauge condition (like the whole shift vector in GR).

Let us consider solutions to (50) in order to check that the constraint is physically acceptable. Notice that proving the existence of a physically solution is crucial for the viability of the generalized unimodular theory of gravity. Integrating $\mathcal{C}_{i}^{N}=0$ gives a first-order PDE as

$$
\begin{gathered}
\sqrt{h}\left[3 F_{1}(h)+2 F_{2}(h)\right] \partial_{i} N_{1}^{i}-\frac{2}{M_{\mathrm{P}}^{2}}\left[f(h) F_{1}(h)\right. \\
\left.-F_{1}^{2}(h)+f(h) F_{2}(h)\right] h_{i j} \pi^{i j}=c_{1},
\end{gathered}
$$

where $c_{1}$ is a constant of integration, which can be rewritten as

$$
\begin{aligned}
\partial_{i} N_{1}^{i}= & \frac{2}{M_{\mathrm{P}}^{2}}\left[\frac{f(h) F_{1}(h)-F_{1}^{2}(h)+f(h) F_{2}(h)}{3 F_{1}(h)+2 F_{2}(h)}\right] \frac{h_{i j} \pi^{i j}}{\sqrt{h}} \\
& +\frac{c_{1}}{\sqrt{h}\left[3 F_{1}(h)+2 F_{2}(h)\right]} .
\end{aligned}
$$

This PDE for the shift vector has the form of a Gauss' law with a complicated source term that depends on the canonical variables $h_{i j}$ and $\pi^{i j}$. Boundary conditions should be chosen to match the assumed physical setting. In general, we can use the corresponding boundary conditions of GR, since the field equations closely resemble those of GR [12]. Fortunately, there is a class of functions $f(h)$ for which the constraint (50) has a much simpler form.

The constraint (50) becomes a homogeneous PDE when the function $f(h)$ is such that $f(h) F_{1}(h)-F_{1}^{2}(h)+$ $f(h) F_{2}(h)=0$, i.e.,

$$
h f(h) f^{\prime \prime}(h)-h\left[f^{\prime}(h)\right]^{2}+f(h) f^{\prime}(h)=0 .
$$

Remarkably, this condition is satisfied by any power-law function

$$
f(h)=\alpha_{n} h^{n},
$$

where the power $n \in \mathbb{R}-\left\{0,-\frac{1}{2}\right\}$ and $\alpha_{n}$ is a fixed scalar density of weight $-2 n$. Since (57) is quadratic in $f$, a power series function $f$ does not generally satisfy it. For example, $f(h)=\alpha_{n} h^{n}+\beta_{m} h^{m}$ satisfies (57) if $m=n$, and hence $f(h)$ reduces to (58). From now on, we shall concentrate the analysis on power-law functions (58). The constraint (50) becomes

$$
\mathcal{C}_{i}^{N}=\frac{1}{\sqrt{h}} \partial_{i}\left(\partial_{j} N^{j} \sqrt{h} \alpha_{n} h^{n}\right) \approx 0,
$$

where we have dropped a finite constant factor $n(2 n+1)$. The integrated form of the condition (56) is written as

$$
\partial_{i} N_{1}^{i}=\frac{c_{1}}{\sqrt{h} \alpha_{n} h^{n}} .
$$

Since $\alpha_{n} h^{n}=f(h) \approx N>1$, the sign of the right-hand side of (60) is set by the sign of the constant $c_{1}$. For $c_{1}>0$ the shift vector field has sources everywhere, while for $c_{1}<0$ there are wells everywhere. The condition (60) takes a particularly simple form if we choose the constant of integration as $c_{1}=0$, since then the divergence of the shift vector vanishes:

$$
\partial_{i} N_{1}^{i}=0
$$

This equation clearly admits a physically viable solution, for example, $N_{1}^{i}=0$.

The consistency of $\mathcal{C}_{i}^{N}$ under time evolution can be ensured by fixing the Lagrange multiplier $v_{N}^{i}$ of the 
constraint $\pi_{i} \approx 0$, since $\mathcal{C}_{i}^{N}$ has a nonvanishing Poisson bracket with $\pi_{i}$, so that $\mathcal{C}_{i}^{N}$ and $\pi_{i} \approx 0$ are second-class constraints. The consistency condition for the constraint (59) is obtained as

$$
\begin{aligned}
\left\{\int_{\Sigma_{t}} d^{3} x \sqrt{h} \xi^{i} \mathcal{C}_{i}^{N}, H\right\} \approx & \int_{\Sigma_{t}} d^{3} x \partial_{i} \xi^{i}\left[-\alpha_{n} h^{n+(1 / 2)} \partial_{j} v_{N}^{j}\right. \\
& +\frac{2 n+1}{M_{\mathrm{P}}^{2}} \partial_{j} N^{j}\left(\alpha_{n} h^{n}\right)^{2} h_{k l} \pi^{k l} \\
& -\left(n+\frac{1}{2}\right) \partial_{j} N^{j}\left(N^{k} \partial_{k} h\right. \\
& \left.\left.+2 \partial_{k} N^{k} h\right) \alpha_{n} h^{n-(1 / 2)}\right],
\end{aligned}
$$

which has to vanish. We used the constraint $\mathcal{C}_{N}$ to write $N \approx \alpha_{n} h^{n}$ after the evaluation of the Poisson bracket. We decompose $v_{N}^{i}$ in the same way as the shift vector (51), since only its divergence appears in the consistency condition:

$$
v_{N}^{i}=v_{N \mid 1}^{i}+v_{N \mid \mathrm{t}}^{i}, \quad \partial_{i} v_{N \mid \mathrm{t}}^{i}=0 .
$$

The consistency condition (62) can be satisfied by solving the longitudinal component of the Lagrange multiplier $v_{N}^{i}$ from the following PDE:

$$
\begin{aligned}
\partial_{i}\left[\alpha_{n} h^{n+(1 / 2)} \partial_{j} v_{N \mid 1}^{j}-\frac{2 n+1}{M_{\mathrm{P}}^{2}} \partial_{j} N_{1}^{j}\left(\alpha_{n} h^{n}\right)^{2} h_{k l} \pi^{k l}\right. \\
\left.\quad+\left(n+\frac{1}{2}\right) \partial_{j} N_{1}^{j}\left(N^{k} \partial_{k} h+2 \partial_{k} N_{1}^{k} h\right) \alpha_{n} h^{n-(1 / 2)}\right]=0 .
\end{aligned}
$$

Together, (50) and (64) form a system of second-order PDEs that should be solved for the shift vector $N^{i}$ and the Lagrange multiplier vector $v_{N}^{i}$. We do not attempt to solve (64), in general, but rather settle for showing that a physically viable solution exist. We can also integrate (64) to obtain a first-order PDE as

$$
\begin{aligned}
& \alpha_{n} h^{n+(1 / 2)} \partial_{j} v_{N \mid 1}^{j}-\frac{2 n+1}{M_{\mathrm{P}}^{2}} \partial_{j} N_{1}^{j}\left(\alpha_{n} h^{n}\right)^{2} h_{k l} \pi^{k l} \\
& \quad+\left(n+\frac{1}{2}\right) \partial_{j} N_{1}^{j}\left(N^{k} \partial_{k} h+2 \partial_{k} N_{1}^{k} h\right) \alpha_{n} h^{n-(1 / 2)}=c_{2},
\end{aligned}
$$

where $c_{2}$ is a constant of integration. When the constants of integration are chosen as $c_{1}=c_{2}=0$ in (60) and (64), we obtain from (65) that the divergence of the longitudinal component of the Lagrange multiplier vector $v_{N}^{i}$ vanishes:

$$
\partial_{i} v_{N \mid 1}^{i}=0
$$

which can be solved for a given boundary condition. The transverse component of the Lagrange multiplier vector $v_{N}^{i}$ is left undetermined (until gauge fixing). This completes the proof that the structure of constraints is consistent under time evolution.

Let us consider a concrete example of boundary conditions and discuss the conditions (60) and (65) further. On an asymptotically flat spacetime, we choose the boundary conditions in asymptotic coordinates as [15]

$$
\begin{aligned}
N & =1+O\left(\frac{1}{r}\right), & N^{i} & =O\left(\frac{1}{r}\right), \\
h_{i j} & =\delta_{i j}+O\left(\frac{1}{r}\right), & \pi^{i j} & =O\left(\frac{1}{r^{2}}\right) .
\end{aligned}
$$

Thus, $\partial_{i} N^{i}$ behaves as $O\left(r^{-2}\right)$ in the asymptotic region, where the asymptotic radial coordinate $r$ is very large. The right-hand side of (60) behaves similarly as $\partial_{i} N^{i}$ when (58) behaves asymptotically as $O\left(r^{2}\right)$. Since $h$ behaves as $1+O\left(r^{-1}\right)$, and hence $h^{n}$ behaves as $1+n O\left(r^{-1}\right)$, in turn $\alpha_{n}$ should exhibit a behavior $O\left(r^{2}\right)$ in the asymptotic region. Then from (65) we see that the Lagrange multiplier $v_{N}^{i}$ must behave as $O\left(r^{-1}\right)$ in the asymptotic region, i.e., in the same manner as the shift vector.

\section{B. Generally noncovariant constraints with spatially nonlocal linear dependence}

Like in conventional unimodular gravity [5], we prefer a single local constraint over the gradient one (47). In both unimodular gravity $[5,16]$ and the local theory of vacuum energy sequestering [17], we can use a technique that decomposes the variables which are involved in the constraints into time-dependent zero modes and spacetimedependent average-free modes whose integral over $\Sigma_{t}$ vanishes. The decomposition enabled a transparent counting and identification of the physical degrees of freedom and a rigorous treatment of the nonlocal linear dependence of the constraints [5,17] according to the BatalinVilkovisky formalism [18]. Unfortunately, that technique does not work well in the present case of generalized unimodular gravity, since the relevant constraints (47) and (59) consist of partial derivatives of scalar densities instead of derivatives of scalars. The reason for the problem can be traced back to the way that the general covariance is broken by the generalized unimodular condition (2).

We shall explain the problem briefly. A scalar field $\phi$, such as $\lambda$, could be decomposed to a time-dependent component and a space-dependent component as

$$
\phi(t, x)=\phi_{0}(t)+\bar{\phi}(t, x)
$$

where the zero mode describes the time-dependent average of $\phi$ over space: 


$$
\phi_{0}(t)=\frac{1}{V_{\Sigma_{t}}} \int_{\Sigma_{t}} d^{3} x \sqrt{h} \phi(t, x), \quad V_{\Sigma_{t}}=\int_{\Sigma_{t}} d^{3} x \sqrt{h}
$$

and the spacetime-dependent component has a vanishing integral over space:

$$
\int_{\Sigma_{t}} d^{3} x \sqrt{h} \bar{\phi}(t, x)=0
$$

Then the spatial derivative $\partial_{i} \phi=\partial_{i} \bar{\phi}$, and hence a constraint $\partial_{i} \phi=0$, would mean that $\bar{\phi}$ is a constant on the spatial hypersurface and the condition (70) would impose that constant to zero $\bar{\phi}=0$. In unimodular gravity [5], this enables us to replace the constraint $\partial_{i} \lambda \approx 0$ with $\bar{\lambda} \approx 0$, leaving the zero mode $\lambda_{0}$ unconstrained. Unfortunately, a scalar density $\rho$, such as $\sqrt{h} F_{1}(h) \lambda$, cannot be decomposed into a constant component and a space-dependent component. Instead, we would have to decompose a scalar density of unit weight as

$\rho=\frac{\sqrt{h}}{V_{\Sigma_{t}}} \rho_{0}+\bar{\rho}, \quad \rho_{0}=\int_{\Sigma_{t}} d^{3} x \rho, \quad \int_{\Sigma_{t}} d^{3} x \bar{\rho}=0$,

so that the integrals are well defined. Now the spatial partial derivative of $\rho$ is written as

$$
\partial_{i} \rho=\partial_{i} \sqrt{h} \frac{\rho_{0}}{V_{\Sigma_{t}}}+\partial_{i} \bar{\rho} .
$$

Therefore, $\partial_{i} \rho=0$ does not imply $\partial_{i} \bar{\rho}=0$. Instead, $\partial_{i} \rho=$ 0 imposes a relation between $\rho_{0}, \partial_{i} \bar{\rho}$, and the metric. Therefore, the constraints (47) and (59) cannot be decomposed in a suitable form with this approach. Note that the problem would not appear if the constraints (47) and (59) involved covariant derivatives, but that is not the case due to the breakdown of general covariance.

Therefore, we need a method for handling constraints of the form $\partial_{i} \rho \approx 0$, which are not generally covariant when $\rho$ is a scalar density on the spatial hypersurface. Our general solution to the problem is based on the introduction of a new variable $q(t)$ that is an arbitrary function of time. The constraint $\partial_{i} \rho \approx 0$ can be replaced with a new constraint $\rho-q \approx 0$. Those two constraints are equivalent, assuming that the variable $q$ is an arbitrary function of time, since the former constraint is invariant under the translation $\rho \rightarrow$ $\rho+\epsilon$ for any $\epsilon(t)$. The time evolution of $q$ is not determined by the equations of motion. This ensures that $q$ is an arbitrary function of time, which carries a single degree of freedom, a so-called zero mode. We shall treat $q(t)$ as an external variable or a background function. Note that the constraints $\partial_{i} \rho \approx 0$ across the spatial hypersurface are linearly dependent, $\int_{\Sigma_{t}} d^{3} x \partial_{i} \rho=0$, since the value of $\rho$ at $x^{i} \rightarrow \pm \infty$ with each $i=1,2,3$ (or at the spatial boundary if one exists) is the same. However, a bonus of the new approach is that the new constraints $\rho-q \approx 0$ are not linearly dependent across the spatial hypersurface, since $q$ is an independent function instead of a component of the decomposition of $\rho$. Thus, while the constraints $\rho-q \approx 0$ clearly imply $\partial_{i} \rho \approx 0$, and vice versa, the former constraints do not share the nonlocal linear dependence of the latter constraints.

We observe that the above method could be used as well when $\rho$ is a scalar, like in unimodular gravity $[5,16]$ and in the local theory of vacuum energy sequestering [17]. In those cases, the advantage of the approach would be to avoid the decomposition of variables and eliminate the nonlocal linear dependence of the constraints. In the present case of generalized unimodular gravity, however, the new approach is a necessity rather than an option.

Now we shall use the above method for the constraint (47), which also leads to a replacement of the secondary constraint (59). We introduce a new variable $q(t)$, which depends only on time. The constraint (47) is replaced with a constraint of the form

$$
\mathcal{C}_{1}=\sqrt{h} F_{1}(h) \lambda-q \approx 0 .
$$

The consistency condition for (73) implies a secondary constraint that replaces (59). It is obtained as $\sqrt{h} f(h) \partial_{i} N^{i} \approx 0$, which can be simplified to define the constraint as

$$
\mathcal{C}_{2}=\partial_{i} N_{1}^{i} \approx 0,
$$

where the decomposition of the shift vector (51) is also used. Observe that (74) already appeared in (61) as a specific solution to the constraint (59). The constraints (73) and (74) do not exhibit the nonlocal linear dependence of the constraints (47) and (59). Now that we have proved that the structure of constraints is consistent under time evolution and written them in a suitable linearly independent form, we proceed to the canonical analysis regarding the physical degrees of freedom of the generalized theory in its different cases.

\section{Counting of physical degrees of freedom}

In the case of a constant function $f(h)$, there are three physical degrees of freedom for each point of space. The extra physical degree of freedom compared to GR is due to the absence of a Hamiltonian constraint for the Hamiltonian (45), since (46) is no longer a constraint. Consequently, there is also a nonvanishing bulk contribution to the Hamiltonian on the constraint surface: $H \approx \int_{\Sigma_{t}} d^{3} x f \mathcal{H}_{T}^{0} \neq 0$. In GR, the Hamiltonian constraint is regarded to fix the conformal factor of the metric $h_{i j}$ [19], which leaves the conformally invariant metric independent. The absence of a Hamiltonian constraint 
in the present case means that the conformal factor of the metric becomes an independent dynamical variable.

In the case of unimodular gravity, $f(h)=\epsilon_{0} / \sqrt{h}$, there are the same two local physical degrees of freedom as in GR and an extra zero mode that describes the cosmological constant [5].

All the remaining choices for $f(h)$ share the same physical degrees of freedom. We can identify the second-class constraints of the theory as $\mathcal{C}_{N}, \pi_{N}, \mathcal{C}_{1}, p_{\lambda}, \mathcal{C}_{2}$, and $\pi_{i \mid 1}$. The first four constraints can be used to eliminate the variables $N, \pi_{N}, \lambda$, and $p_{\lambda}$, while the last two secondclass constraints fix the longitudinal component of the shift vector. The Dirac bracket can be shown to be equivalent to the Poisson bracket for the remaining variables. The Hamiltonian is thus written as

$$
H=\int_{\Sigma_{t}} d^{3} x\left[f(h) \mathcal{H}_{T}^{0}+\left(N_{\mathrm{t}}^{i}+N_{\mathrm{l}}^{i}\right) \mathcal{H}_{i}+v_{N}^{i} \pi_{i \mid \mathrm{t}}\right]
$$

where $N_{1}^{i}$ is the solution to (60) under given boundary conditions, and the Hamiltonian constraint was written as

$$
\begin{aligned}
\mathcal{H}_{T} & =\mathcal{H}_{T}^{0}+\frac{q}{F_{1}(h)} \\
& =\frac{2}{M_{\mathrm{P}}^{2} \sqrt{h}} \pi^{i j} \mathcal{G}_{i j k l} \pi^{k l}-\frac{M_{\mathrm{P}}^{2} \sqrt{h}}{2}{ }^{(3)} R+\frac{q}{F_{1}(h)} \approx 0 .
\end{aligned}
$$

Therefore, in the case of a general function $f(h)$, we have the same two local physical degrees of freedom as in GR and an additional time-dependent variable $q(t)$. The latter is an external zero mode produced by the restriction of general covariance. While the evolution of $q(t)$ is not determined by the equations of motion, it has to be consistent with the boundary conditions and evolution of metric variables, since they are related by the Hamiltonian constraint (76), and the equation of the motion for the momentum $\pi^{i j}$ depends explicitly on $q$. The Hamiltonian on the constraint surface again contains a nonvanishing bulk contribution, but thanks to the constraint (76) it is now given as $H \approx-q \int_{\Sigma_{t}} d^{3} x f(h) / F_{1}(h)$. Next, we discuss how the above bulk terms contribute to the definition of total gravitational energy of generalized unimodular gravity.

\section{Total gravitational energy}

For a given solution, we define the total energy associated with a time translation along $t^{\mu}=N n^{\mu}+N^{\mu}$ as the value of the physical Hamiltonian. We assume that the solution asymptotically approaches a static background solution. Then the physical Hamiltonian is defined as the difference of the Hamiltonian of the solution $H$ and the Hamiltonian of the static background $H_{\mathrm{b}}$ as $H_{\text {phys }}=$ $H-H_{\mathrm{b}}$. In GR, the total gravitational energy is given by boundary terms as [14]

$$
E_{\mathrm{GR}}=-M_{\mathrm{P}}^{2} \int_{\mathcal{B}_{t}} d^{2} x N \sqrt{\sigma}\left({ }^{(2)} K-{ }_{0}^{(2)} K\right)+2 \int_{\mathcal{B}_{t}} d^{2} x N_{i} r_{j} \pi^{i j},
$$

where $\mathcal{B}_{t}$ is the boundary of the spatial hypersurface $\Sigma_{t}$, ${ }^{(2)} K$ is the extrinsic curvature of the boundary, $\sigma$ is the determinant of the metric induced on $\mathcal{B}_{t}$, and $r^{i}$ is the unit normal to $\mathcal{B}_{t}$. The subscript " 0 " denotes the quantities associated with the static background. The total energy for any background can be obtained from the general expression above. That includes the ADM energy for an asymptotically flat spacetime, as well as the total energy for asymptotically anti-de Sitter spacetimes and asymptotically conical spacetimes.

In generalized unimodular gravity, we have shown above that the bulk Hamiltonian contains a nonvanishing contribution on the constraint surface. Physically, this was expected, since the field equation (8) contain an additional stress-energy contribution due to the constraint (2) that breaks down general covariance.

When $f$ is a constant, the physical Hamiltonian contains a nonvanishing bulk contribution:

$$
H_{\mathrm{phys}}=H_{\mathrm{phys}}^{\mathrm{GR}}+f \int_{\Sigma_{t}} d^{3} x\left(\mathcal{H}_{T}^{0}-{ }_{0} \mathcal{H}_{T}^{0}\right) .
$$

Since the momentum ${ }_{0} \pi^{i j}$ vanishes for the static background, we have

$$
{ }_{0} \mathcal{H}_{T}^{0}=-\frac{M_{\mathrm{P}}^{2}}{2} \sqrt{{ }_{0} h_{0}^{(3)}} R
$$

Hence, we obtain the total energy of a given solution as

$$
\begin{aligned}
E= & E_{\mathrm{GR}}+f \int_{\Sigma_{t}} d^{3} x\left(\frac{2}{M_{\mathrm{P}}^{2} \sqrt{h}} \pi^{i j} \mathcal{G}_{i j k l} \pi^{k l}\right. \\
& \left.-\frac{M_{\mathrm{P}}^{2}}{2}\left(\sqrt{h^{(3)}} R-\sqrt{{ }_{0} h_{0}^{(3)}} R\right)\right) .
\end{aligned}
$$

In the general case, when $f(h)$ is not a constant and it does not match the case of unimodular gravity, we obtain the physical Hamiltonian as

$$
H_{\mathrm{phys}}=H_{\mathrm{phys}}^{\mathrm{GR}}-\int_{\Sigma_{t}} d^{3} x\left(q \frac{f(h)}{F_{1}(h)}-q_{0} \frac{f\left({ }_{0} h\right)}{F_{1}\left({ }_{0} h\right)}\right) .
$$

Since the function $q$ of time is not determined by the dynamical equations, we could assume that the solution for it matches the background, $q=q_{0}$. Then the total energy is given as

$$
E=E_{\mathrm{GR}}-q_{0} \int_{\Sigma_{t}} d^{3} x\left(\frac{f(h)}{F_{1}(h)}-\frac{f\left({ }_{0} h\right)}{F_{1}\left({ }_{0} h\right)}\right) .
$$


The bulk contributions to the total energy in both cases are inconvenient, but that appears to be a direct consequence of the given type of violation of general covariance. There is an important exception. For a power-law function $f$ (58), the fraction $f(h) / F_{1}(h)$ is a constant $n^{-1}$, which is independent of $h$, and hence the bulk contribution to the total energy (82) vanishes. Consequently, the total energy of GR (77) is retained in this case.

\section{PATH INTEGRAL}

In order to recognize the differences compared to GR and unimodular gravity at the quantum level, we shall work out the formal canonical path integral for generalized unimodular gravity. The case of unimodular gravity, $f(h)=$ $\epsilon_{0} / \sqrt{h}$, has been analyzed in Ref. [5], and that will serve as a point of comparison for the present generalized version of the theory.

\section{A. Constant function $f$}

When $f$ is a constant, we first integrate over the variables $N, \pi_{N}, \lambda$, and $p_{\lambda}$ by using the second-class constraints $\mathcal{C}_{N}$, $\pi_{N}, \mathcal{H}_{T}$, and $p_{\lambda}$. Then the Hamiltonian appears in the form (45). Gauge-fixing conditions for the first-class constraints $\mathcal{H}_{i}$ and $\pi_{i}$ are introduced as $\chi^{i}$ and $\sigma^{i}=N^{i}-f^{i}$, respectively, and we assume for simplicity that the gauge conditions have vanishing Poisson brackets with each other. Once the shift variable has been integrated, the path integral is obtained as

$$
\begin{aligned}
\mathcal{Z}= & \mathcal{N}_{1} \int \prod_{\chi^{\mu}} \mathcal{D} h_{i j} \mathcal{D} \pi^{i j} \delta\left(\chi^{i}\right) \delta\left(\mathcal{H}_{j}\right)\left|\operatorname{det}\left\{\chi^{i}, \mathcal{H}_{j}\right\}\right| \\
& \times \exp \left[\frac{i}{\hbar} \int d t \int_{\Sigma_{t}} d^{3} x\left(\pi^{i j} \partial_{t} h_{i j}-f \mathcal{H}_{T}^{0}-f^{i} \mathcal{H}_{i}\right)\right] .
\end{aligned}
$$

Using the integral representation $\delta\left(\mathcal{H}_{i}\right) \propto \int \prod_{x^{\mu}} \mathcal{D} N^{i} \times$ $\exp \left(-\frac{i}{\hbar} \int d t \int_{\Sigma_{t}} d^{3} x N^{i} \mathcal{H}_{i}\right)$ and shifting the reintroduced shift variables as $N^{i}+f^{i} \rightarrow N^{i}$, we obtain the path integral as

$$
\begin{aligned}
\mathcal{Z}= & \mathcal{N}_{2} \int \prod_{x^{\mu}} \mathcal{D} N^{i} \mathcal{D} h_{i j} \mathcal{D} \pi^{i j} \delta\left(\chi^{i}\right)\left|\operatorname{det}\left\{\chi^{i}, \mathcal{H}_{j}\right\}\right| \\
& \times \exp \left[\frac{i}{\hbar} \int d t \int_{\Sigma_{t}} d^{3} x\left(\pi^{i j} \partial_{t} h_{i j}-f \mathcal{H}_{T}^{0}-N^{i} \mathcal{H}_{i}\right)\right] .
\end{aligned}
$$

Integration over the momentum $\pi^{i j}$ is performed in the same way as in GR, which gives

$$
\begin{aligned}
\mathcal{Z}= & \mathcal{N}_{3} \int \prod_{\chi^{\mu}} \mathcal{D} N^{i} \mathcal{D} h_{i j} h^{-3 / 2} \delta\left(\chi^{i}\right)\left|\operatorname{det}\left\{\chi^{i}, \mathcal{H}_{j}\right\}\right| \\
& \times \exp \left[\frac{i}{\hbar} \frac{M_{\mathrm{P}}^{2}}{2} \int d t \int_{\Sigma_{t}} d^{3} x f \sqrt{h}\left(K_{i j} \mathcal{G}^{i j k l} K_{k l}+{ }^{(3)} R\right)\right],
\end{aligned}
$$

where $K_{i j}=\frac{1}{2 f}\left(\partial_{t} h_{i j}-2 D_{(i} N_{j)}\right)$. Thus, the two major differences compared to GR remain unaltered at the quantum level. The lapse is fixed to a constant $f$, and there is no Hamiltonian constraint. Therefore, only the functional determinant associated with gauge fixing of the spatial diffeomorphisms is present. We may rewrite the path integral in a form that resembles the covariant path integral of GR by reintroducing the lapse along with the constraint $\mathcal{C}_{N}=$ $N-f=\left(-g^{00}\right)^{-1 / 2}-f$ as

$$
\begin{aligned}
\mathcal{Z}= & \mathcal{N}_{4} \int \prod_{\chi^{\mu}} \mathcal{D} g_{\mu \nu} g^{00}(-g)^{-3 / 2} \delta\left(\left(-g^{00}\right)^{-1 / 2}-f\right) \delta\left(\chi^{i}\right) \\
& \times\left|\operatorname{det}\left\{\chi^{i}, \mathcal{H}_{j}\right\}\right| \exp \left(\frac{i}{\hbar} S_{\mathrm{EH}}\left[g_{\mu \nu}\right]\right)
\end{aligned}
$$

where $S_{\mathrm{EH}}\left[g_{\mu \nu}\right]$ is the Einstein-Hilbert action without a cosmological constant. General covariance is, of course, broken not only due to the constraint $\left(-g^{00}\right)^{-1 / 2}=f$ but also due to absence of the fourth generator $\mathcal{H}_{T}$ of spacetime diffeomorphism. The measure of integration has been written in a gauge-invariant form [20], except for the (gauge) conditions imposed by the $\delta$ functions.

\section{B. General function $\boldsymbol{f}(\boldsymbol{h})$}

In the general case, i.e., when $f^{\prime}(h) \neq 0$ and $3 f^{\prime}(h)+2 h f^{\prime \prime}(h) \neq 0$, we first use the second-class constraints as $\mathcal{C}_{N}, \pi_{N}, \mathcal{C}_{1}, p_{\lambda}, \mathcal{C}_{2}$, and $\pi_{i \mid 1}$ to integrate out the variables $N, \pi_{N}, \lambda, p_{\lambda}, N_{1}^{i}$, and $\pi_{i||}$. Hence, we attain the Hamiltonian (75). Gauge-fixing conditions for the firstclass constraints $\mathcal{H}_{\mu}=\left(\mathcal{H}_{T}, \mathcal{H}_{i}\right)$ and $\pi_{i \mid \mathrm{t}}$ are introduced as $\chi^{\mu}$ and $\sigma_{\mathrm{t}}^{i}=N_{\mathrm{t}}^{i}-f_{\mathrm{t}}^{i}$, respectively, and we assume that the gauge conditions have vanishing Poisson brackets with each other. The pair of constraints $\pi_{i \mid \mathrm{t}}$ and $\sigma_{\mathrm{t}}^{i}$ is used to integrate over $N_{\mathrm{t}}^{i}$ and $\pi_{i \mid \mathrm{t}}$. Now the path integral can be written as

$$
\begin{aligned}
\mathcal{Z}= & \mathcal{N}_{1} \int \prod_{x^{\mu}} \mathcal{D} h_{i j} \mathcal{D} \pi^{i j} \mathcal{D} q \delta\left(\chi^{\mu}\right) \delta\left(\mathcal{H}_{\nu}\right)\left|\operatorname{det}\left\{\chi^{\mu}, \mathcal{H}_{\nu}\right\}\right| \\
& \times \exp \left[\frac { i } { \hbar } \int d t \int _ { \Sigma _ { t } } d ^ { 3 } x \left(\pi^{i j} \partial_{t} h_{i j}-f(h) \mathcal{H}_{T}^{0}\right.\right. \\
& \left.\left.-\left(f_{\mathrm{t}}^{i}+N_{\mathrm{l}}^{i}\right) \mathcal{H}_{i}\right)\right]
\end{aligned}
$$

where one should notice that $N_{1}^{i}$ is the solution to (60) under given boundary conditions. Using the integral representation $\delta\left(\mathcal{H}_{\nu}\right) \propto \int \prod_{x^{\mu}} \mathcal{D} N \mathcal{D} N^{i} \exp \left[-\frac{i}{\hbar} \int d t \int_{\Sigma_{t}} d^{3} x\left(N \mathcal{H}_{T}+N^{i} \mathcal{H}_{i}\right)\right]$ and shifting the reintroduced lapse and shift variables as $N+f(h) \rightarrow N$ and $N^{i}+f_{\mathrm{t}}^{i}+N_{\mathrm{l}}^{i} \rightarrow N^{i}$, we obtain the path integral as 


$$
\begin{aligned}
\mathcal{Z}= & \mathcal{N}_{2} \int \prod_{x^{\mu}} \mathcal{D} N \mathcal{D} N^{i} \mathcal{D} h_{i j} \mathcal{D} \pi^{i j} \mathcal{D} q \delta\left(\chi^{\mu}\right)\left|\operatorname{det}\left\{\chi^{\mu}, \mathcal{H}_{\nu}\right\}\right| \\
& \times \exp \left[\frac { i } { \hbar } \int d t \int _ { \Sigma _ { t } } d ^ { 3 } x \left(\pi^{i j} \partial_{t} h_{i j}-N \mathcal{H}_{T}^{0}-N^{i} \mathcal{H}_{i}\right.\right. \\
& \left.\left.-\frac{q(N-f(h))}{F_{1}(h)}\right)\right] .
\end{aligned}
$$

Finally, we integrate over the momentum $\pi^{i j}$ and the variable $q(t)$, which gives the path integral as

$$
\begin{aligned}
\mathcal{Z}= & \mathcal{N}_{3} \int \prod_{x^{\mu}} \mathcal{D} g_{\mu \nu} g^{00}(-g)^{-3 / 2} \delta\left(\chi^{\mu}\right) N\left|\operatorname{det}\left\{\chi^{\mu}, \mathcal{H}_{\nu}\right\}\right|_{\pi^{i j}[h]} \\
& \times \delta\left(\int_{\Sigma_{t}} d^{3} x \frac{(\sqrt{-g}-\sqrt{h} f(h))}{\sqrt{h} F_{1}(h)}\right) \exp \left(\frac{i}{\hbar} S_{\mathrm{EH}}\left[g_{\mu \nu}\right]\right),
\end{aligned}
$$

where $\pi^{i j}[h]=\frac{M_{\mathrm{P}}^{2}}{2} \sqrt{h} \mathcal{G}^{i j k l} K_{k l}$ and $S_{\mathrm{EH}}\left[g_{\mu \nu}\right]$ is the EinsteinHilbert action without a cosmological constant. The above integration measure has again been written in a gaugeinvariant form [20]. The difference compared to GR is the integrated condition on the metric in the measure, which imposes an integral of the generalized unimodular condition (2) over the spatial hypersurfaces to be satisfied as

$$
\int_{\Sigma_{t}} d^{3} x \frac{(\sqrt{-g}-\sqrt{h} f(h))}{\sqrt{h} F_{1}(h)}=0 .
$$

That is, the metric in the path integral has to satisfy the generalized unimodular condition (2) in average, weighted with $\sqrt{h} F_{1}(h)$, over each spatial hypersurface. In unimodular gravity [5], we have a similar integrated condition, $\int_{\Sigma_{t}} d^{3} x\left(\sqrt{-g}-\epsilon_{0}\right)=0$, but without a weighting factor.

\section{PROPAGATION OF PERTURBATIONS IN THE CASE OF A CONSTANT FUNCTION $\boldsymbol{f}(\boldsymbol{h})$}

In order to elucidate the nature of the extra physical degree of freedom found in the case of a constant $f$, we consider a linearization of the theory. In particular, we obtain the field equations for weak perturbations of the metric induced on the spatial hypersurfaces and study the propagation of perturbations in vacuum.

We consider a background spacetime with a metric of the form

$$
g_{\mu \nu} d x^{\mu} d x^{\nu}=-d t^{2}+h_{i j} d x^{i} d x^{j}
$$

Any metric can be written to this form in Gaussian normal coordinates, but such coordinates usually cover only a part of spacetime. We set the constant function $f$ to 1 , and we choose to gauge fix the shift vector as $N^{i}=0$.
The action for the partially gauge-fixed system is thus written as

$$
\begin{aligned}
S & =\int d t \int_{\Sigma_{t}} d^{3} x\left(\pi^{i j} \partial_{t} h_{i j}-\mathcal{H}_{T}^{0}\right)+S_{\mathrm{m}} \\
& =\frac{M_{\mathrm{P}}^{2}}{2} \int d t \int_{\Sigma_{t}} d^{3} x\left(\frac{1}{4} \mathcal{G}^{i j k l} \partial_{t} h_{i j} \partial_{t} h_{k l}+{ }^{(3)} R\right)+S_{\mathrm{m}},
\end{aligned}
$$

where $S_{\mathrm{m}}$ is the action for matter. Recall that, in the case of a constant $f$, the system was shown to be symmetric only under diffeomorphisms on the spatial hypersurface, since the Hamiltonian constraint served only to fix the value of the auxiliary variable $\lambda$. Hence, there is an extra physical degree of freedom, which is carried by the spatial metric $h_{i j}$. The field equations for $h_{i j}$ are obtained by varying $h^{i j}$ as

$$
\begin{gathered}
\partial_{t}\left(\left(\delta_{i}^{k} \delta_{j}^{l}-h_{i j} h^{k l}\right) \partial_{t} h_{k l}\right)+\partial_{t} h_{i k} \partial_{t} h_{j l} h^{k l} \\
-\partial_{t} h_{i j} \partial_{t} h_{k l} h^{k l}+2^{(3)} R_{i j}=2 M_{\mathrm{P}}^{-2} T_{i j},
\end{gathered}
$$

where $T_{i j}=-\frac{2}{\sqrt{h}} \frac{\delta S_{m}}{\delta h^{i j}}$ is the stress tensor for matter.

Then the metric induced on the spatial hypersurfaces is expanded as

$$
h_{i j}={ }_{0} h_{i j}+\gamma_{i j}, \quad\left|\gamma_{i j}\right| \ll 1,
$$

where ${ }_{0} h_{i j}$ is the spatial background metric that satisfies the field equations for a given distribution of matter. The inverse of the metric is $h^{i j}={ }_{0} h^{i j}-\gamma^{i j}$, where $\gamma^{i j}=$ ${ }_{0} h^{i k}{ }_{0} h^{j l} \gamma_{k l}$. The linearized field equations are obtained as

$$
\begin{gathered}
\partial_{t}^{2}\left(\gamma_{i j}-{ }_{0} h_{i j} \gamma\right)+{ }_{0} D^{k}{ }_{0} D_{i} \gamma_{j k}+{ }_{0} D^{k}{ }_{0} D_{j} \gamma_{i k} \\
-{ }_{0} D^{2} \gamma_{i j}-{ }_{0} D_{i 0} D_{j} \gamma=\left(2 M_{\mathrm{P}}^{-2}\right) \mathcal{T}_{i j},
\end{gathered}
$$

where $\gamma={ }_{0} h^{i j} \gamma_{i j}$ and ${ }_{0} D_{i}$ is the covariant derivative determined by the spatial background metric ${ }_{0} h_{i j}$, we denote ${ }_{0} D^{i}={ }_{0} h^{i j}{ }_{0} D_{j}$ and ${ }_{0} D^{2}={ }_{0} h^{i j}{ }_{0} D_{i 0} D_{j}$, and $\mathcal{T}_{i j}$ represents perturbation of matter fields. We decompose the perturbation of the spatial metric to a traceless component $s_{i j}$ and the trace component $\gamma$ as

$$
\gamma_{i j}=s_{i j}+\frac{1}{3}{ }_{0} h_{i j} \gamma
$$

The traceless component can further be decomposed to a transverse (divergence-free) component and a longitudinal component ${ }^{4}$ :

$$
s_{i j}=\gamma_{i j}^{\mathrm{TT}}+\gamma_{i j}^{\mathrm{L}},
$$

\footnotetext{
${ }^{4}$ Alternatively, $\gamma_{i j}$ could be first decomposed to transverse and longitudinal components, and then the trace of the transverse component could be separated. In other words, the longitudinal component could be defined with or without a trace. See $[21,22]$ for details.
} 
where

$$
{ }_{0} D^{j} \gamma_{i j}^{\mathrm{TT}}=0, \quad \gamma_{i j}^{\mathrm{L}}={ }_{0} D_{i} W_{j}+{ }_{0} D_{j} W_{i}-\frac{2}{3}{ }_{0} h_{i j 0} D_{k} W^{k} .
$$

The linearized field equations are rewritten as

$$
\begin{gathered}
{ }_{0} \square s_{i j}-{ }_{0} D_{0}^{k} D_{i} s_{j k}-{ }_{0} D_{0}^{k} D_{j} s_{i k}+\frac{2}{3}{ }_{0} h_{i j} \partial_{t}^{2} \gamma+\frac{1}{3}{ }_{0} h_{i j 0} D^{2} \gamma \\
+\frac{1}{3}{ }_{0} D_{i 0} D_{j} \gamma=\left(2 M_{\mathrm{P}}^{-2}\right) \mathcal{T}_{i j},
\end{gathered}
$$

where ${ }_{0} \square=-\partial_{t}^{2}+{ }_{0} D^{2}$ is the d'Alembertian.

We are interested in the propagation of gravitational perturbations in vacuum; i.e., we take $\mathcal{T}_{i j}=0$. For that purpose, it is appropriate to consider the background to be the Minkowski spacetime, ${ }_{0} h_{i j}=\delta_{i j}$. The linearized field equations (99) are then written as

$$
\begin{aligned}
& \square s_{i j}-\partial^{k} \partial_{i} s_{j k}-\partial^{k} \partial_{j} s_{i k}+\frac{2}{3} \delta_{i j} \partial_{t}^{2} \gamma+\frac{1}{3} \delta_{i j} \partial^{k} \partial_{k} \gamma \\
& \quad+\frac{1}{3} \partial_{i} \partial_{j} \gamma=0,
\end{aligned}
$$

where $\partial^{i}=\delta^{i j} \partial_{j}$ and $\square=-\partial_{t}^{2}+\partial^{i} \partial_{i}$ is the d'Alembertian in Minkowski spacetime. In order to fix the symmetry under spatial diffeomorphisms, we consider two possible gauge conditions. First, we choose the transverse coordinate condition

$$
\partial^{j} s_{i j}=0,
$$

which fixes the longitudinal component:

$$
\partial^{j} \gamma_{i j}^{\mathrm{L}}=\partial^{j} \partial_{j} W_{i}+\frac{1}{3} \partial_{i} \partial^{j} W_{j}=0 .
$$

The field equations simplify to

$$
\square \gamma_{i j}^{\mathrm{TT}}+\frac{2}{3} \delta_{i j} \partial_{t}^{2} \gamma+\frac{1}{3} \delta_{i j} \partial^{k} \partial_{k} \gamma+\frac{1}{3} \partial_{i} \partial_{j} \gamma=0 .
$$

The traceless transverse mode and the trace mode are still coupled due to the last term. In GR, the trace component $\gamma$ is not dynamical, since it is determined by the 00 component of the Einstein equation as $\partial^{i} \partial_{i} \gamma=\frac{1}{3} \partial^{i} \partial^{j} s_{i j}$, so that its appearance in (103) is not a complication. However, here the trace mode is dynamical, and hence we prefer to decouple the dynamical equations for $s_{i j}$ and $\gamma$.

For that purpose, the most elucidating gauge choice is the harmonic coordinate condition on the spatial hypersurface

$$
\partial^{j} \gamma_{i j}=\frac{1}{2} \partial_{i} \gamma
$$

which is written for the traceless component as

$$
\partial^{j} s_{i j}=\frac{1}{6} \partial_{i} \gamma
$$

i.e., the longitudinal component is determined by the trace component as

$$
\partial^{j} \gamma_{i j}^{\mathrm{L}}=\partial^{j} \partial_{j} W_{i}+\frac{1}{3} \partial_{i} \partial^{j} W_{j}=\frac{1}{6} \partial_{i} \gamma .
$$

We emphasize that our harmonic coordinate condition (104) is not the usual harmonic or Lorentz condition of linearized GR. The present coordinate condition (104) is defined on the spatial hypersurface, so that the coordinates satisfy $h^{i j} D_{i} D_{j} x^{k}=0$. Now the field equations (100) for the traceless and trace modes are decoupled as

$$
\begin{gathered}
\square s_{i j}=0, \\
\partial_{t}^{2} \gamma+\frac{1}{2} \partial^{i} \partial_{i} \gamma=0 .
\end{gathered}
$$

The traceless mode satisfies the standard wave equation, and these perturbations travel at the speed of light. These are the usual gravitational waves. The trace mode, however, satisfies an elliptic PDE in spacetime, which is highly unusual in physics. Elliptic PDEs are common in space but not in spacetime.

\section{A. Dynamics of the trace mode}

Since the dynamics of the traceless mode is determined by the usual wave equation, we now focus on the unusual elliptic form of equation for the trace mode. The dynamical equation for the trace mode (108) resembles the Laplace equation in four-dimensional Euclidean space, except that the equation is anisotropic with respect to time and space due to the factor $\frac{1}{2}$. The elliptic nature of the equation means that the trace mode does not propagate in the usual sense, but rather it spreads out from the source in a peculiar way.

We can solve the elliptic PDE (108) with conventional methods, for example, via the separation of variables. Consider an ansatz of the form

$$
\gamma=A(t) B(x) .
$$

The PDE is separated as

$$
\begin{aligned}
& \frac{d^{2} A}{d t^{2}}-\frac{k^{2}}{2} A=0, \\
& \triangle B+k^{2} B=0,
\end{aligned}
$$

where $k^{2}$ is a separation constant and $\triangle=\partial^{i} \partial_{i}$ is the spatial Laplacian. Both equations are of a familiar type and easy to solve with boundary conditions chosen to match the physical situation, in particular, the shape and symmetry of the perturbation, which is related to the nature of the matter source. When $k^{2}>0$, the general solution to (110) is 


$$
A(t)=c_{1} e^{k t / \sqrt{2}}+c_{2} e^{-k t / \sqrt{2}}
$$

The PDE for $B$ is the Helmholtz equation in three-dimensional space, i.e., the same one obtained for the wave equation, which can be solved by the separation of variables in several coordinate systems.

Consider a plane perturbation that travels in the direction of one of the Cartesian coordinates $x^{i}$, so that $B$ depends only on one of the spatial coordinates. Hence, the spatial equation (111) becomes one dimensional, and it has the general solution

$$
B(x)=c_{3} \sin (k x)+c_{4} \cos (k x) .
$$

Hence, in this case, the full solution for the trace perturbation reads

$$
\gamma(t, x)=\sum_{k}\left(c_{1} e^{k t / \sqrt{2}}+c_{2} e^{-k t / \sqrt{2}}\right)\left(c_{3} \sin (k x)+c_{4} \cos (k x)\right) .
$$

In order to obtain a specific solution, we need to specify suitable initial and/or boundary conditions on $\gamma$. For example, we could impose initial conditions as

$$
\gamma(0, x)=f_{0}(x), \quad \partial_{t} \gamma(0, x)=f_{1}(x),
$$

where $f_{0}$ and $f_{1}$ are functions such that $\left|f_{0}\right| \ll 1$ and $\left|f_{1}\right| \ll 1$ everywhere. Furthermore, boundary conditions could be imposed in the spatial direction as well, for instance, a Dirichlet boundary condition

$$
\gamma(t,-L)=\gamma(t, L)=b(t) .
$$

As an example, we consider solutions that satisfy the following initial conditions for a given $k$ :

$$
\begin{gathered}
\gamma(0, x)=2 a \sin (k x), \\
\partial_{t} \gamma(0, x)=\sqrt{2} b k \sin (k x),
\end{gathered}
$$

where $a$ and $b$ are dimensionless constants that satisfy $|b| \leq|a| \ll 1$, and the boundary conditions are defined as

$$
\gamma(t,-L)=\gamma(t, L)=0 .
$$

The solution is obtained as

$\gamma(t, x)=\sum_{n=1}^{\infty}\left(\left(a_{n}+b_{n}\right) e^{k_{n} t / \sqrt{2}}+\left(a_{n}-b_{n}\right) e^{-k_{n} t / \sqrt{2}}\right) \sin \left(k_{n} x\right)$,

where $\sum_{n=1}^{\infty}\left|a_{n}\right| \ll 1, \quad\left|b_{n}\right| \leq\left|a_{n}\right|, \quad k_{n}=\frac{n \pi}{L}, \quad n \in \mathbb{Z}_{+}$.

The time-dependent factor of the perturbation (119) for each $k_{n}$ is a sum of an exponentially increasing term and an exponentially decreasing term. Given enough time, the exponentially increasing term will begin to dominate, which happens for a given $k_{n}$ when $t>\left(\sqrt{2} k_{n}\right)^{-1} \ln \left(\frac{a_{n}-b_{n}}{a_{n}+b_{n}}\right)$. The only way to avoid the exponential growth of the perturbation with time is to fine-tune the initial conditions by setting $b_{n}$ (extremely close) to $-a_{n}$. When $b_{n}=-a_{n}$, the perturbation diminishes exponentially with time. That kind of evolution would, however, require an especially fine-tuned source to produce the perturbation. For general initial conditions, the perturbation will eventually begin to grow exponentially. An exponential growth of a perturbation with time is a sign of an instability. When the initial and boundary conditions are consistent with a negative separation constant, $k^{2}<0$, the roles of time and space are interchanged, and hence the plane perturbation would generally grow exponentially with the distance in space, once the distance is large enough.

The linearized description is valid only as long as the perturbation remains small, $|\gamma| \ll 1$. For a perturbation (119) that consists of a single mode $k_{n}$, that implies the time must be small enough to satisfy $\left|a_{n}+b_{n}\right| e^{k_{n} t / \sqrt{2}} \ll 1$, or $t \ll-\sqrt{2} k_{n}^{-1} \ln \left|a_{n}+b_{n}\right|$ by at least one order of magnitude. Beyond that, the linearization of the system is invalid, and hence a nonperturbative treatment would become necessary.

The length scales $k_{n}^{-1}$ that are present in a perturbation are comparable to the scales involved in the source that produces the perturbation. For any observation of gravitational waves, the length scales $k_{n}^{-1}$ involved in the perturbation are very small compared to the distance, in space and time, between the source and the observer. Thus, unless the initial conditions are fine-tuned, the exponentials in (119) are very large, and hence $k_{n} t$ is expected to be greater than $\ln \left(\frac{a_{n}-b_{n}}{a_{n}+b_{n}}\right)$. Therefore, the perturbation increases exponentially with time. Similar results can be obtained for spherical and cylindrical perturbations, where for $k^{2}>0$ the radial dependence of the perturbation is given by the (spherical) Bessel functions. We conclude that, while the trace mode and the traceless mode are decoupled in the chosen gauge, and therefore the usual gravitational wave solutions for the traceless perturbation are unaltered, the trace perturbation has been shown to grow exponentially with time, which implies that the trace mode is unstable. On the other hand, for initial and boundary conditions that are consistent with a negative separation constant, $k^{2}<0$, a plane perturbation would be oscillatory in time, but it would grow exponentially with the distance in space. 


\section{CONCLUSIONS}

We have studied the Hamiltonian formalism and path integral quantization of generalized unimodular gravity, where general covariance is broken by imposing the determinant of the metric of spacetime equal to a function of the determinant of the spatial metric (2). We emphasized that there are two ways to look at the theory. Those different points of view are analogous to the case of unimodular [23], where the field equation for the metric is either the traceless Einstein equation or (thanks to the Bianchi identity) the Einstein equation with a cosmological constant. In the first approach, we can eliminate the field $\lambda$ that is used to impose the generalized unimodular constraint, since it is nondynamical and determined by the Hamiltonian constraint (30) or, equivalently, by the projection (16) of the modified Einstein equation. This approach is aligned with the interpretation that $\lambda$ is a (nondynamical) variable of the gravitational sector. Alternatively, after the field equations or the canonical equations of motion have been derived, one can begin to regard $\lambda$ as the energy density of an extra matter component. Then $\lambda$ could be treated as an independent matter component. We have used the first approach in our Hamiltonian analysis, so that $\lambda$ is treated as a gravitational variable throughout the analysis.

The physical content of the model for a general function $f(h)$ resembles the case of (customary) unimodular gravity. Both theories contain two local physical degrees of freedom, which correspond to the graviton and an additional zero mode. In the generalized model, however, the zero mode is not fixed to a constant dynamically, which differs from unimodular gravity, where the constant value of the zero mode is the cosmological constant [5]. Instead, the Hamiltonian constraint of the generalized model contains a bulk term that depends on time and on the determinant of the spatial metric (76). That also results in the presence of a nonvanishing bulk term in the physical Hamiltonian (81). The corresponding bulk contribution to the total energy (82) was shown to vanish for a power-law function $f$ (58), so that the definition of total energy matches the one of GR. This enhances the prospects of models with a power-law function, in addition to the fact that the constraint (50) is simplified greatly for such functions.

Particular attention was paid to the special case of a constant function $f$, where an extra degree of freedom is found in each point of space. This interesting consequence of a constant $f$ was shown in the Hamiltonian analysis, and already predicted in a careful treatment of the modified Einstein field equations (8), and it is clearly visible in the canonical path integral of the theory. The presence of the extra degree of freedom can be traced to the fact that the Hamiltonian constraint (30) is no longer a first-class constraint but rather a second-class constraint that determines the variable $\lambda$. The appearance of the local extra degree of freedom is the result of a breakdown of general covariance down to diffeomorphism invariance on the spatial hypersurface. That was also shown to imply that the Hamiltonian contains a nonvanishing bulk contribution on the constraint surface, which contributes to the definition of total gravitational energy.

In order to further analyze the implications of the extra degree of freedom in the case of a constant $f$, we have considered the propagation of perturbations in a vacuum. When the background is chosen as Minkowski spacetime, and the perturbation of the spatial metric is decomposed in terms of a traceless component $s_{i j}$ and trace component $\gamma$, it was found that the traceless mode satisfies a standard wave equation, which corresponds to the usual gravitational waves, while the trace mode satisfies an elliptic PDE in spacetime (108), showing that this mode does not propagate as a wave, but rather it spreads out in spacetime. Examining a solution to this equation for a given set of initial and boundary conditions, it was shown that the trace mode is oscillatory in space but behaves exponentially with time (or vice versa, depending on the sign of the value of the separation constant, which is determined by the boundary and/or initial conditions). Then the trace mode was shown to grow exponentially with time, when enough time has passed. That could be avoided only by fine-tuning the initial conditions. Hence, the trace mode is generally unstable on the Minkowski background.

Another point that deserves attention was our proposal of handling constraints that impose a vanishing gradient, i.e., $\partial_{i} \phi \approx 0$, in favor of local constraints in the Hamiltonian analysis for the case of a general function $f(h)$. The usual approach is to decompose the variable $\phi$ into a timedependent zero mode $\phi_{0}$ and a spacetime-dependent average-free mode $\bar{\phi}$ [16], so that the above constraint is replaced with $\bar{\phi} \approx 0$. Both constraints exhibit a nonlocal linear dependence, since their integrals over the spatial hypersurface vanish, and hence the constraint must be handled according to the formalism of Ref. [18]. This decomposition enables a clear identification of the physical degrees of freedom in both unimodular gravity and the local theory of vacuum energy sequestering. Unfortunately, this technique does not work in the present case of generalized unimodular gravity, since the relevant constraints (47) and (59) consist of partial derivatives of scalar densities instead of derivatives of scalars.

Our solution to the problem circumvents the need to perform a decomposition, but rather it is based on the introduction of a new (nondynamical) variable $q(t)$ that is an arbitrary function of time, carrying a single physical degree of freedom, a so-called zero mode, so that the constraint $\partial_{i} \rho \approx 0$ is replaced with a new constraint $\rho-q \approx 0$. Those two constraints are equivalent assuming that the variable $q$ is an arbitrary function of time. The second major difference is that in this approach the new constraints $\rho-q \approx 0$ are no longer linearly dependent across the spatial hypersurface, since $q$ is an independent 
function instead of a component of the decomposition of $\rho$. Hence, in this approach, the quantization of generalized unimodular theory did not require the treatment of BatalinVilkovisky formalism, and the usual canonical path integral could be used.

Gravitational theories that violate general covariance are rather rare for good reasons. This has particularly been the case since generally covariant formulations of unimodular gravity were created [9] (see also [2,5,16]). The action of generalized unimodular gravity (4) does not admit a generally invariant formulation via reparametrization of coordinates due to the presence of the function $f(h)$. Thus, the generalized unimodular theory is a truly noncovariant modification of GR. One area where nonrelativistic gravity has been particularly fruitful is Hořava-Lifshitz gravity [10], where general covariance is sacrificed at high energies in order to achieve power-counting renormalizability without introducing ghosts. As in generalized unimodular gravity, the violation of general covariance in HořavaLifshitz gravity implies the presence of an extra scalar degree of freedom. The extra mode is well behaved in the current formulation of the theory [24]. It is also possible to eliminate the extra scalar by either extending the symmetry of the theory [25] or introducing additional constraints [26]. Naturally, such additions are not useful in generalized unimodular gravity, since removing the extra degree of freedom would defeat the purpose of the proposal, which is the extra fluid element. We emphasize that the Lorentz violation in generalized unimodular gravity takes place at all energy scales, which is particularly problematic at low energies, since that may conflict with observed bounds on Lorentz violation. A scrutiny of phenomenological viability is clearly required.

\section{ACKNOWLEDGMENTS}

M. O. gratefully acknowledges support from the Emil Aaltonen Foundation. R. B. acknowledges partial support from Conselho Nacional de Desenvolvimento Científico e Tecnológico (CNPq Project No. 304241/2016-4) and Fundação de Amparo à Pesquisa do Estado de Minas Gerais (FAPEMIG Project No. APQ-01142-17).
[1] W. Buchmuller and N. Dragon, Einstein gravity from restricted coordinate invariance, Phys. Lett. B 207, 292 (1988).

[2] A. Padilla and I. D. Saltas, A note on classical and quantum unimodular gravity, Eur. Phys. J. C 75, 561 (2015).

[3] Y.J. Ng and H. van Dam, Possible Solution to the Cosmological Constant Problem, Phys. Rev. Lett. 65, 1972 (1990).

[4] Y. J. Ng and H. van Dam, Unimodular theory of gravity and the cosmological constant, J. Math. Phys. (N.Y.) 32, 1337 (1991).

[5] R. Bufalo, M. Oksanen, and A. Tureanu, How unimodular gravity theories differ from general relativity at quantum level, Eur. Phys. J. C 75, 477 (2015).

[6] S. Weinberg, The cosmological constant problem, Rev. Mod. Phys. 61, 1 (1989).

[7] N. Kaloper and A. Padilla, Sequestering the Standard Model Vacuum Energy, Phys. Rev. Lett. 112, 091304 (2014).

[8] N. Kaloper, A. Padilla, D. Stefanyszyn, and G. Zahariade, Manifestly Local Theory of Vacuum Energy Sequestering, Phys. Rev. Lett. 116, 051302 (2016).

[9] M. Henneaux and C. Teitelboim, The cosmological constant and general covariance, Phys. Lett. B 222, 195 (1989).

[10] P. Hořava, Quantum gravity at a Lifshitz point, Phys. Rev. D 79, 084008 (2009).
[11] A. H. Chamseddine and V. Mukhanov, Mimetic dark matter, J. High Energy Phys. 11 (2013) 135.

[12] A. O. Barvinsky and A. Y. Kamenshchik, Darkness without dark matter and energy-Generalized unimodular gravity, Phys. Lett. B 774, 59 (2017).

[13] R. L. Arnowitt, S. Deser, and C. W. Misner, in Gravitation: An Introduction to Current Research, edited by L. Witten (Wiley, New York, 1962), p. 227; Republished in Republication of: The dynamics of general relativity, Gen. Relativ. Gravit. 40, 1997 (2008).

[14] S. W. Hawking and G. T. Horowitz, The gravitational Hamiltonian, action, entropy and surface terms, Classical Quantum Gravity 13, 1487 (1996).

[15] T. Regge and C. Teitelboim, Role of surface integrals in the Hamiltonian formulation of general relativity, Ann. Phys. (N.Y.) 88, 286 (1974).

[16] K. V. Kuchar, Does an unspecified cosmological constant solve the problem of time in quantum gravity?, Phys. Rev. D 43, 3332 (1991).

[17] R. Bufalo, J. Klusoň, and M. Oksanen, Canonical formulation and path integral for local vacuum energy sequestering, Phys. Rev. D 94, 044005 (2016).

[18] I. A. Batalin and G. A. Vilkovisky, Quantization of gauge theories with linearly dependent generators, Phys. Rev. D 28, 2567 (1983).

[19] N. O'Murchadha and J. W. York, Initial-value problem of general relativity. 1. General formulation and physical interpretation, Phys. Rev. D 10, 428 (1974). 
[20] E.S. Fradkin and G. A. Vilkovisky, S matrix for gravitational field. II. Local measure, general relations, elements of renormalization theory, Phys. Rev. D 8, 4241 (1973).

[21] S. Deser, Covariant decomposition and the gravitational Cauchy problem, Ann. I. H. P.: Phys. Theor. 7, 149 (1967).

[22] J. W. York, Jr., Covariant decompositions of symmetric tensors in the theory of gravitation, Ann. I. H. P.: Phys. Theor. 21, 319 (1974).
[23] W. G. Unruh, Unimodular theory of canonical quantum gravity, Phys. Rev. D 40, 1048 (1989).

[24] D. Blas, O. Pujolas, and S. Sibiryakov, Consistent Extension of Horava Gravity, Phys. Rev. Lett. 104, 181302 (2010).

[25] P. Horava and C. M. Melby-Thompson, General covariance in quantum gravity at a Lifshitz point, Phys. Rev. D 82, 064027 (2010).

[26] M. Chaichian, J. Kluson, and M. Oksanen, Nonprojectable Horava-Lifshitz gravity without the unwanted scalar graviton, Phys. Rev. D 92, 104043 (2015). 